\title{
Multifractal Structure of the Divergence Points of Some Homogeneous Moran Measures
}

\author{
JiaQing Xiao and YouMing He \\ Wuhan College, Zhongnan University of Economics and Law, Wuhan 430070, China \\ Correspondence should be addressed to JiaQing Xiao; xiaojq@whut.edu.cn \\ Received 18 January 2014; Accepted 3 March 2014; Published 7 April 2014 \\ Academic Editor: Christian Maes
}

Copyright (C) 2014 J. Xiao and Y. He. This is an open access article distributed under the Creative Commons Attribution License, which permits unrestricted use, distribution, and reproduction in any medium, provided the original work is properly cited.

\begin{abstract}
The point $x$ for which the $\operatorname{limit}_{r \rightarrow 0}(\log \mu(B(x, r)) / \log r)$ does not exist is called divergence point. Recently, multifractal structure of the divergence points of self-similar measures has been investigated by many authors. This paper is devoted to the study of some Moran measures with the support on the homogeneous Moran fractals associated with the sequences of which the frequency of the letter exists; the Moran measures associated with this kind of structure are neither Gibbs nor self-similar and than complex. Such measures possess singular features because of the existence of so-called divergence points. By the box-counting principle, we analyze multifractal structure of the divergence points of some homogeneous Moran measures and show that the Hausdorff dimension of the set of divergence points is the same as the dimension of the whole Moran set.
\end{abstract}

\section{Introduction and Statement of Results}

1.1. Moran Set. Let $\left\{n_{k}\right\}_{k \geq 1}$ be a sequence of positive integers and let $\left\{r_{k}\right\}$ be a sequence of positive real number with $n_{k} r_{k}<$ 1 for any $k \in \mathbb{N}$. Define $D_{o}=\phi$, and for any $k \geq 1$, set $D_{m k}=$ $\left\{\left(i_{m}, i_{m+1}, \ldots, i_{k}\right) ; 1 \leq i_{j} \leq n_{j}, m \leq j \leq k\right\}, D_{k}=D_{1, k}$, and

$$
D=\bigcup_{k \geq 0} D_{k}
$$

If $\sigma=\left(\sigma_{1}, \sigma_{2}, \ldots, \sigma_{k}\right) \in D_{k}, \tau=\left(\tau_{1}, \ldots, \tau_{m-k}\right) \in D_{k+1, m}$, let $\sigma * \tau=\left(\sigma_{1}, \ldots, \sigma_{k}, \tau_{1}, \ldots, \tau_{m-k}\right)$. And for $1 \leq l \leq k$, remark $\sigma \mid l=\left(\sigma_{1}, \ldots, \sigma_{l}\right)$.

Definition 1. Suppose $J$ is a closed interval of length 1 . The collection $\mathscr{F}=\left\{J_{\sigma} ; \sigma \in D\right\}$ of closed subintervals of $J$ is said to have a homogeneous Moran structure, if it satisfies the following conditions (MSC):

(i) $J_{\phi}=J$;

(ii) for all $k \geq 0$ and $\sigma \in D_{k}, J_{\sigma * 1}, J_{\sigma * 2}, \ldots, J_{\sigma * n_{k+1}}$ are subintervals of $J_{\sigma}$ and satisfy that $J_{\tau * i}^{\circ} \cap J_{\tau * j}^{\circ}=$ $\phi(i \neq j)$, where $A^{\circ}$ denotes the interior of $A$; (iii) for any $k \geq 1$ and $\sigma \in D_{k-1}, 1 \leq j \leq n_{k}$,

$$
\frac{\left|J_{\sigma * j}\right|}{\left|J_{\sigma}\right|}=r_{k}
$$

where $|A|$ denotes the diameter of $A$.

Suppose that $\mathscr{F}$ is a collection of closed subintervals of $J$ having homogeneous Moran structure, and set

$$
E_{k}=\bigcup_{\sigma \in D_{k}} J_{\sigma}, \quad E=\bigcap_{k \geq 0} E_{k} .
$$

It is ready to see that $E$ is a nonempty compact set. The set $E:=E(\mathscr{F})$ is called the homogeneous Moran set associated with the collection $\mathscr{F}$.

Let $\mathscr{F}_{k}=\left\{J_{\sigma} ; \sigma \in D_{k}\right\}$, and let $\mathscr{F}=\bigcup_{k>0} \mathscr{F}_{k}$. The elements of $\mathscr{F}_{k}$ are called the basic elements of order $k$ of the homogeneous Moran set $E$ and the elements of $\mathscr{F}$ are called the basic elements of the homogeneous Moran set $E$.

Remark 2. If $\lim _{n \rightarrow \infty} \sup _{\sigma \in D_{n}}\left|J_{\sigma}\right|>0$, then $E$ contains interior points. Thus, the measure and dimension properties will be trivial. We assume therefore that

$$
\lim _{n \rightarrow \infty} \sup _{\sigma \in D_{n}}\left|J_{\sigma}\right|=0 \text {. }
$$


Proposition 3 (see [1, Proposition 3.1]). For a homogeneous Moran set $E$ defined as above, suppose furthermore that

$$
\lim _{k \rightarrow \infty} \frac{\log r_{k}}{\log r_{1} r_{2} \cdots r_{k-1}}=0
$$

Then we have

$$
\operatorname{dim}_{H} E=\liminf _{k \rightarrow \infty} s_{k}
$$

where $s_{k}$ satisfies the equation $\sum_{\sigma \in D_{k}} r_{\sigma}^{s_{k}}=1$ for each $k$.

Let $A=\left\{a_{1}, a_{2}, \ldots, a_{m}\right\}$, and let $\omega=s_{1} s_{2} \cdots s_{k} \cdots$ be a sequence over $A, s_{i} \in A$. For $k \geq 1$, write $\omega_{k}=\left.\omega\right|_{k}=$ $s_{1} s_{2} \cdots s_{k}$; then $\left|\omega_{k}\right|=k$. We denote by $\left|\omega_{k}\right|_{a_{i}}$ the number of occurrences of the letter $a_{i}$ in $\omega_{k}$. If for any $a_{i} \in A$, $\lim _{k \rightarrow \infty}\left(\left|\omega_{k}\right|_{a_{i}} / k\right)=\eta_{i}>0$, then we say that the sequence $\omega$ has the frequency vector $\eta=\left(\eta_{1}, \eta_{2}, \ldots, \eta_{m}\right)$. It is easy to see that $\sum_{i=1}^{m}\left|\omega_{k}\right|_{a_{i}}=k$ and $\sum_{j=1}^{m} \eta_{j}=1$. For $\eta=\left(\eta_{1}, \eta_{2}, \ldots, \eta_{m}\right)$, let

$$
A_{\eta}^{\mathbb{N}}=\left\{\omega=\left\{s_{k}\right\}_{k \geq 1} ; s_{k} \in A, \lim _{k \rightarrow \infty} \frac{\left|\omega_{k}\right|_{a_{i}}}{k}=\eta_{i}, 1 \leq i \leq m\right\} .
$$

For $1 \leq i \leq m$, let $m_{i} \in \mathbb{N}$ and let $c_{i}$ be a positive real number with $m_{i} c_{i} \leq 1$. For $\omega \in A^{\mathbb{N}}$, in the homogeneous Moran construction above, for any $k \geq 1$ if $s_{k}=a_{i}$ take $n_{k}=m_{i}, r_{k}=c_{i}$. Then we construct the homogeneous Moran set relating to $\omega \in A_{\eta}^{\mathbb{N}}$ and denote it by $E(\omega)=\left\{J,\left\{n_{k}\right\},\left\{r_{k}\right\}\right\}$.

Remark 4. In this paper, we assume that $J_{\sigma} \in \mathscr{F}_{k}(k \geq 1)$, let $J_{\sigma * 1}, J_{\sigma * 2}, \ldots, J_{\sigma * n_{k+1}}$ be the $n_{k+1}$ basic intervals of order $k+1$ contained in $J_{\sigma}$ arranged from left to right. For all $1 \leq j \leq$ $n_{k+1}-1$, let $d\left(J_{\sigma * j}, J_{\sigma *(j+1)}\right) \geq \Delta_{k}\left|J_{\sigma}\right|$, where $\left\{\Delta_{k}\right\}$ is a sequence of positive real number. Let $\Delta=\inf _{k \geq 1} \Delta_{k}$. In this paper we suppose $\Delta>0$.

1.2. Moran Measure. Let $P_{a_{i}}=\left(p_{i 1} p_{i 2} \cdots p_{i m_{i}}\right)(1 \leq i \leq m)$ be probability vectors; that is, $p_{i j}>0$ and $\sum_{j=1}^{m_{i}} p_{i j}=1(1 \leq$ $i \leq m)$. For any $k \geq 1, \sigma \in D_{k}$, from Section 1.1, we know $\sigma=\sigma_{1} \sigma_{2} \cdots \sigma_{k} \in D_{k}$ where $\sigma_{k} \in\left\{1,2, \ldots, m_{i}\right\}$, if $s_{k}=a_{i}$. For $\sigma=\sigma_{1} \sigma_{2} \cdots \sigma_{k}$, define $\sigma\left(a_{i}\right)$ as follows: let $\omega_{k}=s_{1} \cdots s_{k}$, $e_{1}<e_{2}<\cdots e_{\left|\omega_{k}\right|_{a_{j}}}$ be the occurrences of the letter $a_{i}$ in $\omega_{k}$; then $\sigma\left(a_{i}\right)=\sigma_{e_{1}} \sigma_{e_{2}} \cdots \sigma_{e_{\left|\omega_{k}\right|_{a_{i}}}}$. For convenience we will write $\sigma\left(a_{i}\right)=\sigma_{i_{1}} \sigma_{i_{2}} \cdots \sigma_{i_{\left|\omega_{k}\right|_{a_{i}}}}$, where $\sigma_{i_{j}} \in\left\{1,2, \ldots, m_{i}\right\}(1 \leq i \leq m)$. In fact, $\sigma_{\left(a_{1}\right)} * \sigma_{\left(a_{2}\right)} * \cdots * \sigma_{\left(a_{m}\right)}$ is a rearrangement of $\sigma=$ $\sigma_{1} \cdots \sigma_{k}$. We make the convention that $\sigma_{\left(a_{i}\right)}=\phi$ if $\left|\omega_{k}\right|_{a_{i}}=0$.

Now define

$$
p_{\sigma\left(a_{i}\right)}=p_{i \sigma_{i_{1}}} \cdots p_{i \sigma_{i_{\left|\omega_{k}\right| a_{i}}}}, \quad 1 \leq i \leq m .
$$

It is obvious that $\sum_{\sigma \in D_{k}} \prod_{i=1}^{m} p_{\sigma\left(a_{i}\right)}=1$ for any $k \geq 1$. We make the convention that $p_{\sigma\left(a_{i}\right)}=1$ if $\sigma\left(a_{i}\right)=\phi$.

Let $\mu$ be a mass distribution on $E(\omega)$, such that for any $J_{\sigma} \in \mathscr{F}_{k}, \sigma \in D_{k}$,

$$
\mu\left(J_{\sigma}\right)=p_{\sigma\left(a_{1}\right)} p_{\sigma\left(a_{2}\right)} \cdots p_{\sigma\left(a_{m}\right)},
$$

and $\mu\left(\sum_{\sigma \in D_{k}} J_{\sigma}\right)=1$. Since $\mu$ is related to $\omega$, we denote it by $\mu(\omega)$. Here $\mu(\omega)$ is a homogeneous Moran measure on $E(\omega)$, and it is an extension of the self-similar measure by Hutchinson [2].

1.3. Main Results. From now on, we assume that $E(\omega)$ is a homogeneous Moran fractal defined in Section 1.1, and $\mu(\omega)$ is a probability measure introduced in Section 1.2. The notationa $D, D_{k}, J_{\sigma}, \mu\left(J_{\sigma}\right), P_{\sigma\left(a_{i}\right)}(1 \leq i \leq m)$ are as above; in the following, if $\sigma \in D_{k}, \tau \in D_{k+1, k+n}$, let $r_{\sigma}=\left|J_{\sigma}\right|, p_{\sigma}=\mu\left(J_{\sigma}\right)$ and $r_{\tau}=\left|J_{\sigma * \tau}\right| /\left|J_{\sigma}\right|, p_{\tau}=\mu\left(J_{\sigma * \tau}\right) / \mu\left(J_{\sigma}\right)$. Now we define an auxiliary function $\beta(q)$ as follows. For each $q \in \mathbb{R}$ and $k \geq 1$, there is a unique number $\beta_{k}(q)$ such that

$$
\sum_{\sigma \in D_{k}} p_{\sigma}^{q} r_{\sigma}^{\beta_{k}(q)}=1
$$

By simple calculation, we get

$$
\lim _{k \rightarrow \infty} \beta_{k}(q)=\beta(q)=\frac{\sum_{i=1}^{m} \eta_{i} \log \left(\sum_{j=1}^{m_{i}} p_{i j}^{q}\right)}{\sum_{i=1}^{m} \eta_{i} \log c_{i}} .
$$

Proposition 5 (see [3, Proposition 2.3]). For all $q \in \mathbb{R}, \beta(q)$ defined by (10) satisfies the following:

(i) $\beta(0)=\operatorname{dim} E$;

(ii) $\beta(q)$ is strictly decreasing, and $\lim _{q \rightarrow \mp \infty} \beta(q)= \pm \infty$;

(iii) $\beta(q)$ is convex in $q$, and $\beta(q)$ is strictly convex if and only if $\log p_{i j} / \log c_{i}$ is not the same for all $1 \leq j \leq m_{i}$, $i=1,2, \ldots, m$.

Let $\mu$ be a Borel probability measure on $\mathbb{R}^{d}$; let

$$
\Theta(q ; r)=\sup \sum_{i} \mu\left(B\left(x_{i}, r\right)\right)^{q}, \quad r>0, q \in \mathbb{R},
$$

where the supremum is taken over all families of disjoint closed balls $\left\{B\left(x_{i}, r\right)\right\}_{i}$ with $x_{i} \in \operatorname{supp} \mu$. If $\lim _{r \rightarrow 0}(\log \Theta(q ; r) / \log r)$ exists, we call that the $L^{q}$-spectrum $\tau(q)$ of $\mu$ exists; that is,

$$
\tau(q)=\lim _{r \rightarrow 0} \frac{\log \Theta(q ; r)}{-\log r} .
$$

Peres and Solomyak [4] give alternative definition of $L^{q}$-spectrum. Let $\mu$ be a Borel probability measure on $\mathbb{R}^{d}$. Let $\mathbf{D}_{n}$ be the partition of $\mathbb{R}^{d}$ into grid boxes $\prod_{i=1}^{d}\left[k_{i} 2^{-n},\left(k_{i}+\right.\right.$ $1) 2^{-n}$ ) with $k_{i} \in \mathbb{Z}$. For $q>0$, denote $\tau_{n}^{(q)}(\mu)=\sum_{Q \in \mathbf{D}_{n}}(\mu Q)^{q}$. If $\lim _{n \rightarrow 0}\left(\log \tau_{n}^{(q)}(\mu) /-n \log 2\right)$ exists, we call that the $L^{q}$-spectrum $\tau_{0}(q)$ of $\mu$ exists; that is,

$$
\tau_{0}(q)=\lim _{n \rightarrow 0} \frac{\log \tau_{n}^{(q)}(\mu)}{-n \log 2} .
$$

Peres and Solomyak [4] prove that $\tau(q)=\tau_{0}(q)$.

Proposition 6 (see [5]). Let $\mu$ be a Moran measure supported on the homogeneous Moran fractals $E$; then

$$
\tau(q)=\tau_{0}(q)=\beta(q) .
$$


The Legendre transform of $\beta$ is the function $\beta^{*}$ : $\left(\alpha_{\min }, \alpha_{\max }\right) \rightarrow \mathbb{R}$ defined by

$$
\beta^{*}(\alpha)=\inf _{q \in \mathbb{R}}\{\beta(q)+\alpha q\},
$$

where $\alpha_{\min }=\inf _{q \in \mathbb{R}} \beta^{\prime}(q), \alpha_{\max }=\sup _{q \in \mathbb{R}} \beta^{\prime}(q)$.

Let $X$ be a complete separable metric space and $\mu$ a finite Borel measure on $X$. In the multifractal analysis one is interested in the size of the following level sets:

$$
\begin{array}{r}
X_{\alpha}=\left\{x \in X: \lim _{r \rightarrow 0} \frac{\log \mu(B(x, r))}{\log r}=\alpha\right\}, \\
-\infty \leq \alpha \leq+\infty .
\end{array}
$$

The space $X$ has the following natural decomposition:

$$
X=\bigcup_{-\infty \leq \alpha \leq+\infty} X_{\alpha} \cup X^{o}
$$

where

$$
X^{o}=\left\{x \in X: \lim _{r \rightarrow 0} \frac{\log \mu(B(x, r))}{\log r} \text { does not exist }\right\} .
$$

The set $X^{o}$ is called the set of divergence points and the point $x$ for which the $\operatorname{limit} \lim _{r \rightarrow 0}(\log \mu(B(x, r)) / \log r)$ does not exist is called divergence point. Recently, multifractal structure of the divergence points of self-similar measures has been investigated by a large number of authors. Barreira and Schmeling [6] and Chen and Xiong [7] have shown that for self-similar measures satisfying the SSC the set of divergence points typically has the Hausdorff dimension as the support $K$. Furthermore, Olsen and Winter [8] analyse its structure and give a decomposition of this set for the case that the SSC satisfies. However, with only the OSC satisfied, we cannot do most of the work on a symbolic space and then transfer the results to the subsets of $\mathbb{R}^{d}$, which makes things more difficult. By the box-counting principle we (2011) [9] show that the set of divergence point has still the same Hausdorff dimension as the support $K$ for selfsimilar measures satisfying the OSC. Li et al. [10] further analyse its structure and give a decomposition of this set for the case that the OSC satisfies. This paper is devoted to the study of some Moran measures with the support on the homogeneous Moran fractals associated with the sequences of which the frequency of the letter exists; such measures possess singular features because of the existence of so-called divergence points. By the box-counting principle, we analyze Multifractal structure of the divergence points of some homogeneous Moran measures and show that the Hausdorff dimension of the set of divergence points is the same as the dimension of the whole Moran set. It should be pointed out that the Moran measures associated with this kind of structure are neither Gibbs nor self-similar and more than complex.

Theorem 7. Let $\mu$ be a Moran measure supported on the homogeneous Moran fractals $E(\Omega)$ associated with the sequences $\Omega$ of which the frequency of the letter exists as above. Set

$$
\begin{aligned}
E_{q_{1}, q_{2}}=\{x \in E(\Omega) & \\
\mid \beta^{\prime}\left(q_{1}\right) & =\liminf _{r \rightarrow 0} \frac{\log \mu(B(x, r))}{\log r} \\
< & \left.\limsup _{r \rightarrow 0} \frac{\log \mu(B(x, r))}{\log r}=\beta^{\prime}\left(q_{2}\right)\right\} .
\end{aligned}
$$

Then

$$
\operatorname{dim} E_{q_{1}, q_{2}}=\beta^{*}\left(\beta^{\prime}\left(q_{1}\right)\right) .
$$

By Theorem 7 and Proposition 5, we easily obtain that the Hausdorff dimension of the set of divergence points is the same as the dimension of the whole Moran set.

\section{Several Lemma}

Lemma 8. Suppose that $\alpha \in \mathbb{R}$ is such that $\alpha=-\beta^{\prime}(q)$ for some $q \in \mathbb{R}$. Then for any $\delta>0, \rho>0$,

(I) there exist $d \in(0, \rho), \ell \geq d^{-\beta^{*}(\alpha)+\delta(q+1)}$, an integral number $N$, and $u_{1}, \ldots, u_{\ell} \in D_{N}$ satisfying the following properties:
(a) $d^{1+\delta} \leq r_{u_{i}} \leq d^{1-\delta}$ for all $1 \leq i \leq \ell$,
(b) $d^{\alpha+\delta} \leq p_{u_{i}} \leq d^{\alpha-\delta}$ for all $1 \leq i \leq \ell$;

(II) there exist an integral number $n_{0}$ such that for any integer $k>n_{0}$, there exist $d \in(0, \rho), \ell \geq d^{-\beta^{*}(\alpha)+\delta(q+1)}$, an integral number $N$, and $u_{1}, \ldots, u_{\ell} \in D_{k+1, N+1}$ satisfying the following properties:
(a) $d^{1+\delta} \leq r_{u_{i}} \leq d^{1-\delta}$ for all $1 \leq i \leq \ell$;
(b) $d^{\alpha+\delta} \leq p_{u_{i}} \leq d^{\alpha-\delta}$ for all $1 \leq i \leq \ell$.

Proof. For the given $\delta>0$, we choose a small $0<\epsilon<1$ such that

$$
\begin{aligned}
& \left(\alpha-\frac{\delta}{2}\right) \epsilon \leq \beta(q)-\beta(q+\epsilon) \leq\left(\alpha+\frac{\delta}{2}\right) \epsilon, \\
& \left(\alpha-\frac{\delta}{2}\right) \epsilon \leq \beta(q-\epsilon)-\beta(q) \leq\left(\alpha+\frac{\delta}{2}\right) \epsilon .
\end{aligned}
$$

Using (10), we can pick $0<\gamma<\min \{\epsilon \delta / 6,1\}$ and an integral number $n_{0}$ such that for any integer $n>n_{0}$,

$$
\begin{gathered}
r_{1} r_{2} \cdots r_{n}<\min \left\{\rho, 3^{-1 / \gamma}\right\}, \\
r_{\sigma}^{-\beta(q)+\gamma} \leq \sum_{\sigma \in D_{n}} p_{\sigma}^{q} \leq r_{\sigma}^{-\beta(q)-\gamma}, \\
r_{\sigma}^{-\beta(q+\epsilon)+\gamma} \leq \sum_{\sigma \in D_{n}} p_{\sigma}^{q+\epsilon} \leq r_{\sigma}^{-\beta(q+\epsilon)-\gamma} .
\end{gathered}
$$


Set

$$
\begin{gathered}
\mathbb{B}_{1}=\left\{\sigma \in D_{n}: p_{\sigma} \geq r_{\sigma}^{\alpha-\delta}\right\}, \quad \mathbb{B}_{2}=\left\{\sigma \in D_{n}: p_{\sigma} \geq r_{\sigma}^{\alpha-\delta}\right\}, \\
\mathbb{B}_{3}=\left\{\sigma \in D_{n}: r_{\sigma}^{\alpha+\delta}<p_{\sigma}<r_{\sigma}^{\alpha-\delta}\right\} .
\end{gathered}
$$

Then

$$
\sum_{\sigma \in \mathbb{B}_{1}} p_{\sigma}^{q}=\sum_{\sigma \in \mathbb{B}_{1}} p_{\sigma}^{q+\epsilon} p_{\sigma}^{-\epsilon} \leq r_{\sigma}^{-\beta(q+\epsilon)-\gamma-\epsilon(\alpha-\delta)} \leq r_{\sigma}^{-\beta(q)+\epsilon \delta / 2-\gamma} .
$$

Similarly, we have

$$
\sum_{\sigma \in \mathbb{B}_{2}} p_{\sigma}^{q}=\sum_{\sigma \in \mathbb{B}_{2}} p_{\sigma}^{q-\epsilon} p_{\sigma}^{\epsilon} \leq r_{\sigma}^{-\beta(q-\epsilon)-\gamma+\epsilon(\alpha+\delta)} \leq r_{\sigma}^{-\beta(q)+\epsilon \delta / 2-\gamma} .
$$

These two inequalities together with (22)-(26) imply

$$
\begin{aligned}
\sum_{\sigma \in \mathbb{B}_{3}} p_{\sigma}^{q} & =\left(\sum_{\sigma \in D_{n}}-\sum_{\sigma \in \mathbb{B}_{1}}-\sum_{\sigma \in \mathbb{B}_{2}}\right) p_{\sigma}^{q} \\
& \geq r_{\sigma}^{-\beta(q)+\gamma}-2 r_{\sigma}^{-\beta(q)+\epsilon \delta / 2-\gamma} \\
& =r_{\sigma}^{-\beta(q)+2 \gamma}\left(r_{\sigma}^{-\gamma}-2 r_{\sigma}^{(\epsilon \delta / 2)-3 \gamma}\right) \\
& \geq r_{\sigma}^{-\beta(q)+2 \gamma}\left(r_{\sigma}^{-\gamma}-2\right) \quad\left(\text { by } 0<\gamma<\min \left\{\frac{\epsilon \delta}{6}, 1\right\}\right) \\
& \geq r_{\sigma}^{-\beta(q)+2 \gamma} \quad(\text { by }(24)) .
\end{aligned}
$$

Note that for each $\sigma \in \mathbb{B}_{3}, p_{\sigma} \leq \max \left\{r_{\sigma}^{(\alpha \pm \delta) q}\right\}=r_{\sigma}^{\alpha q-|\delta| q}=$ $\left(r_{1} r_{2} \cdots r_{n}\right)^{\alpha q-|\delta| q}$. Hence,

$$
\sum_{\sigma \in \mathbb{B}_{3}} p_{\sigma}^{q} \leq\left(\# \mathbb{B}_{3}\right)\left(r_{1} r_{2} \cdots r_{n}\right)^{\alpha q-|\delta| q}
$$

which combining with (30) yields

$$
\begin{aligned}
\# \mathbb{B}_{3} & \geq\left(r_{1} r_{2} \cdots r_{n}\right)^{-\beta(q)-\alpha q+\delta|q|+2 \gamma} \\
& =\left(r_{1} r_{2} \cdots r_{n}\right)^{-\beta^{*}(\alpha)+\delta|q|+2 \gamma} \geq\left(r_{1} r_{2} \cdots r_{n}\right)^{-\beta^{*}(\alpha)+\delta(|q|+1)} .
\end{aligned}
$$

We suppose $N$ is an integral number satisfying $N>n_{0}$ and $d=r_{1} r_{2} \cdots r_{N}$. This completes the proof of (I).
Next we prove (II). Note that $\sum_{\sigma \in D_{k}} p_{\sigma}^{q} r_{\sigma}^{\beta_{k}(q)}=1$; we get

$\beta_{k}(q)$

$$
\begin{aligned}
& =\frac{\log \sum_{\sigma \in D_{k}} p_{\sigma}^{q}}{-\log r_{\sigma}} \\
& =\frac{\sum_{i=1}^{m} \eta_{i} \log \sum_{j=1}^{m_{i}} p_{i j}^{q}+\sum_{i=1}^{m}\left(\left(\left|\omega_{k}\right|_{\alpha_{i}} / k\right)-\eta_{i}\right) \log \sum_{j=1}^{m_{i}} p_{i j}^{q}}{-\left(\sum_{i=1}^{m} \eta_{i} \log c_{i}+\sum_{i=1}^{m}\left(\left(\left|\omega_{k}\right|_{\alpha_{i}} / k\right)-\eta_{i}\right) \log c_{i}\right)},
\end{aligned}
$$

$\frac{\log \sum_{\sigma \in D_{k+1, k+n}} p_{\sigma}^{q}}{-\log r_{\sigma}}$

$=\left(\sum_{i=1}^{m} \eta_{i} \log \sum_{j=1}^{m_{1}} p_{i j}^{q}\right.$

$+\sum_{i=1}^{m}\left(\left((k+n)\left(\left(\left|\omega_{k+n}\right|_{\alpha_{i}} /(k+n)\right)-\eta_{i}\right)\right.\right.$

$$
\left.\left.-k\left(\left(\left|\omega_{k}\right|_{\alpha_{i}} / k\right)-\eta_{i}\right)\right) \times(n)^{-1}\right)
$$

$$
\left.\times \log \sum_{j=1}^{m_{i}} p_{i j}^{q}\right)
$$

$\times\left(-\left(\sum_{i=1}^{m} \eta_{i} \log c_{i}\right.\right.$

$$
\begin{aligned}
& +\sum_{i=1}^{m}\left(\left((k+n)\left(\left(\left|\omega_{k+n}\right|_{\alpha_{i}} /(k+n)\right)-\eta_{i}\right)\right.\right. \\
& \left.\left.-k\left(\left(\left|\omega_{k}\right|_{\alpha_{i}} / k\right)-\eta_{i}\right)\right) \times(n)^{-1}\right) \\
& \left.\left.\times \log c_{i}\right)\right)^{-1} .
\end{aligned}
$$

Note that $\lim _{n \rightarrow \infty} \beta_{n}(q)=\beta(q)$ and $\lim _{k \rightarrow \infty}\left(\left|\omega_{k}\right|_{a_{i}} / k\right)=\eta_{i}$ for $1 \leq i \leq m$ and using (33), we can choose an integral $N_{1}$ such that for any integral $k, n>N_{1}$, the following properties are satisfied; that is,

$$
\begin{gathered}
\beta(q)-\gamma \leq \frac{\sum_{i=1}^{m} \eta_{i} \log \sum_{j=1}^{m_{i}} p_{i j}^{q}}{-\sum_{i=1}^{m} \eta_{i} \log c_{i}} \leq \beta(q)+\gamma, \\
\frac{\sum_{i=1}^{m} \eta_{i} \log \sum_{j=1}^{m_{i}} p_{i j}^{q}}{-\sum_{i=1}^{m} \eta_{i} \log c_{i}}-\gamma \leq \frac{\log \sum_{\sigma \in D_{k+1, k+n}} p_{\sigma}^{q}}{-\log r_{\sigma}} \\
\leq \frac{\sum_{i=1}^{m} \eta_{i} \log \sum_{j=1}^{m_{i}} p_{i j}^{q}}{-\sum_{i=1}^{m} \eta_{i} \log c_{i}}+\gamma .
\end{gathered}
$$

Combining (34) we get

$$
\beta(q)-2 \gamma \leq \frac{\log \sum_{\sigma \in D_{k+1, k+n}} p_{\sigma}^{q}}{-\log r_{\sigma}} \leq \beta(q)+2 \gamma .
$$


Therefore

$$
r_{\sigma}^{-(\beta(q)-2 \gamma)} \leq \sum_{\sigma \in D_{k+1, k+n}} p_{\sigma}^{q}<r_{\sigma}^{-(\beta(q)+2 \gamma)} .
$$

Using the same method, we can choose an integral $N_{2}$ such that for any integral $k, n>N_{2}$,

$$
r_{\sigma}^{-(\beta(q+\epsilon)-2 \gamma)} \leq \sum_{\sigma \in D_{k+1, k+n}} p_{\sigma}^{q+\epsilon} \leq r_{\sigma}^{-(\beta(q+\epsilon)+2 \gamma)} .
$$

Set $n_{0}=\max \left\{N_{1}, N_{2}\right\}$, and (36) and (37) satisfy simultaneously. We suppose $N$ is an integral number satisfying $N>n_{0}$ and

$$
\begin{gathered}
\left(\prod_{i=1}^{m}\left(c_{i}^{\eta_{i}}\right)\right)^{N}<\rho, \\
\prod_{i=1}^{m}\left(c_{i}^{\eta_{i}}\right)^{\delta} \leq \prod_{i=1}^{m} c_{i}^{\left((k+N)\left(\left(\left|\omega_{k+N}\right|_{\alpha_{i}} /(k+N)\right)-\eta_{i}\right)-k\left(\left(\left|\omega_{k}\right|_{\alpha_{i}} / k\right)-\eta_{i}\right)\right) / N} \\
\leq \prod_{i=1}^{m}\left(c_{i}^{\eta_{i}}\right)^{-\delta} .
\end{gathered}
$$

Set $d=\left(\prod_{i=1}^{m} c_{i}^{\eta_{i}}\right)^{N}$. Replace (25) and (26) with (36) and (37); we can prove (II) by the same method with (I).

Lemma 9. Let $\mu$ be a Moran measure supported on the homogeneous Moran fractals $E(\Omega)$ associated with the sequences $\Omega$ of which the frequency of the letter exists as above. Set

$$
\begin{aligned}
E_{q_{1}, q_{2}}=\{x \in E(\Omega) \\
\mid \beta^{\prime}\left(q_{1}\right)=\liminf _{r \rightarrow 0} \frac{\log \mu(B(x, r))}{\log r} \\
\left.\quad<\limsup _{r \rightarrow 0} \frac{\log \mu(B(x, r))}{\log r}=\beta^{\prime}\left(q_{2}\right)\right\} .
\end{aligned}
$$

Then

$$
\operatorname{dim} E_{q_{1}, q_{2}} \leq \beta^{*}\left(\beta^{\prime}\left(q_{1}\right)\right) \text {. }
$$

Proof. Let $f=\operatorname{dim} E_{q_{1}, q_{2}}$, then for any $0<\varepsilon<f$, $\mathbf{H}^{f-\varepsilon}\left(E_{q_{1}, q_{2}}\right)=\infty$; Using (39), there exist $E_{q_{1}, q_{2}}^{\circ} \subset E_{q_{1}, q_{2}}$ and a number sequence $\left\{r_{i}\right\} \uparrow 0$ such that

$$
\begin{gathered}
\mathbf{H}^{f-\varepsilon}\left(E_{q_{1}, q_{2}}^{\circ}\right)>1, \\
\mu\left(B\left(x, r_{i}\right)\right) \geq 3^{1 / q_{1}} r_{i}^{\beta^{\prime}\left(q_{1}\right)+\varepsilon},
\end{gathered}
$$

for any $x \in E_{q_{1}, q_{2}}^{\circ}$. We can choose $0<\delta \leq(1 / 2) r_{0}$ such that $\mathbf{H}_{\delta}^{f-\varepsilon}\left(E_{q_{1}, q_{2}}^{\circ}\right) \geq 1$. For any $r_{i} \leq \delta$ and $2^{-\left(n_{i}+1\right)} \leq r_{i}<2^{-n_{i}}$, we consider grid boxes $\left[k 2^{-n_{i}},(k+1) 2^{-n_{i}}\right)$; there exist 3 adjacent grid boxes $A, A_{1}, A_{2}$ such that $B\left(x, r_{i}\right) \subset A \cup A_{1} \cup A_{2}$, and there $x \in A$ and $A_{1}, A_{2}$ are neighbours with $A$. Therefore there exists $A_{0} \in\left\{A, A_{1}, A_{2}\right\}$ such that $\mu\left(A_{0}\right) \geq r_{i}^{\beta^{\prime}\left(q_{1}\right)+\varepsilon} \geq$ $2^{-n_{i}\left(\beta^{\prime}\left(q_{1}\right)+\varepsilon\right)}$ (by $\left.\beta^{\prime}\left(q_{1}\right)<0\right)$. On the other hand, notice that
$\#\left\{C \in \mathbf{D}_{n_{i}} \mid C \cap E_{q_{1}, q_{2}}^{\circ} \neq \varnothing\right\} \geq 2^{-n_{i}(\varepsilon-f)} \mathbf{H}_{\delta}^{f-\varepsilon}\left(E_{q_{1}, q_{2}}^{\circ}\right) \geq 2^{-n_{i}(\varepsilon-f)}$ (by (41)). Thus

$$
\#\left\{C \in \mathbf{D}_{k} \mid \mu\left(A_{0}\right) \geq 2^{-n_{i}\left(\beta^{\prime}\left(q_{1}\right)+\varepsilon\right)}\right\} \geq \frac{1}{3} 2^{-n_{i}(\varepsilon-f)} .
$$

Thus

$$
\tau_{n_{i}}^{\left(q_{1}\right)}(\mu)=\sum_{Q \in \mathbf{D}_{n_{i}}}(\mu Q)^{q_{1}} \geq 2^{-n_{i} q_{1}\left(\beta^{\prime}\left(q_{1}\right)+\varepsilon\right)} 2^{-n_{i}(\varepsilon-f)} .
$$

Therefore

$$
\lim _{i \rightarrow \infty} \frac{\log \tau_{n_{i}}^{\left(q_{1}\right)}(\mu)}{-\log 2^{n_{i}}} \geq-q_{1}\left(\beta^{\prime}\left(q_{1}\right)+\varepsilon\right)+f-\varepsilon .
$$

Using (14) and (15), notice that $\lim _{n \rightarrow \infty}\left(\log \tau_{n}^{\left(q_{1}\right)}(\mu) /-\right.$ $\left.\log 2^{n}\right)=\beta\left(q_{1}\right)$; we have

$$
f \leq \beta\left(q_{1}\right)+q_{1}\left(\beta^{\prime}\left(q_{1}\right)+\varepsilon\right)+\varepsilon .
$$

Thus

$$
\operatorname{dim} E_{q_{1}, q_{2}} \leq \beta^{*}\left(\beta^{\prime}\left(q_{1}\right)\right)
$$

\section{Proof of Theorem 7}

Lemma 10. Suppose $\Delta>0$ ( $\Delta$ is from Remark 4$)$, for all $r>0$, and $x \in J_{\sigma} \in F$, and choose $k, l \in \mathbb{N}$ such that

$$
\begin{gathered}
\left|J_{\sigma \mid k+1}(x)\right| \leq r<\left|J_{\sigma \mid k}(x)\right|, \\
\Delta\left|J_{\sigma \mid l+1}(x)\right| \leq r<\Delta\left|J_{\sigma \mid l}(x)\right|,
\end{gathered}
$$

where $J_{\sigma \mid l+1}(x)$ denote the basic elements of order $l+1$ that contains the point $x$. Then

$$
\begin{aligned}
& \text { (i) } J_{\sigma \mid k+1}(x) \subseteq B(x, r), E \cap B(x, r) \subseteq J_{\sigma \mid l+1}(x), \\
& \text { (ii) } \exists \Delta_{0}>0 \text { such that } k-l \leq \Delta_{0} .
\end{aligned}
$$

Proof. It is obvious that $J_{\sigma \mid k+1}(x) \subseteq B(x, r)$. Now let $y \in E \cap$ $B(x, r)$, but $y \bar{\epsilon} J_{\sigma \mid l+1}(x)$; then there exists $j<l+1$ such that $\tau|j=\sigma| j$, and $\tau|j+1 \neq \sigma| j+1$, and $x \in J_{\sigma \mid j+1}(x), y \in J_{\tau \mid j+1}(y)$. Therefore,

$$
\begin{aligned}
|y-x| & \geq d\left(J_{\tau \mid j+1}(x), J_{\sigma \mid j+1}(y)\right) \\
& \geq \Delta_{j}\left|J_{\sigma \mid j}(x)\right| \geq \Delta\left|J_{\sigma \mid l}(x)\right|>r,
\end{aligned}
$$

which is a contradiction since $y \in E \cap B(x, r)$; therefore $E \cap$ $B(x, r) \subseteq J_{\sigma \mid l+1}(x)$.

Since $\Delta<1$ and $k \geq l$. (48) imply that

$$
1 \leq \frac{\left|J_{\sigma \mid k}(x)\right|}{\Delta\left|J_{\sigma \mid l+1}(x)\right|} \leq \frac{\left(\max \left\{c_{i}\right\}\right)^{k-l-1}}{\Delta},
$$

which yields

$$
k-l \leq 1+\frac{\log \Delta}{\operatorname{logmax}\left\{c_{i}\right\}} \triangleq \Delta_{0} .
$$


Proof of Theorem 7. For any $q_{2}<q_{1}$, define a number sequence $\left\{\alpha_{i}\right\}_{i=1}^{\infty}$ in the following manner:

$$
\begin{gathered}
q_{i}= \begin{cases}q_{1}, & i \text { is odd number; } \\
q_{2}, & i \text { is even number, }\end{cases} \\
\alpha_{i}= \begin{cases}\beta^{\prime}\left(q_{1}\right), & i \text { is odd number; } \\
\beta^{\prime}\left(q_{2}\right), & i \text { is even number. }\end{cases}
\end{gathered}
$$

We choose a positive sequence $\left\{\delta_{i}\right\}_{i=1}^{\infty} \downarrow 0$. For $\left\{\alpha_{i}\right\}$ and $\left\{\delta_{i}\right\}$, there exist an integral number sequence $\left\{n_{0}^{(i)}\right\}_{i=1}^{\infty}\left(n_{0}^{(i)}\right.$ corresponds to $n_{0}$ in Lemma 8$)$ and a real number $\left\{d_{i}\right\}_{i=1}^{\infty}(1>$ $\left.d_{1}>d_{2}>\cdots\right)$ such that the follow properties are satisfied.

For $\alpha_{1}=\beta^{\prime}\left(q_{1}\right)$ and $\delta_{1}$, using Lemma 8 we can pick $u_{11}^{(1)}, u_{12}^{(1)}, \ldots, u_{1 \ell^{(1)}}^{(1)} \in D_{N^{(1)}}, u_{21}^{(1)}, u_{22}^{(1)}, \ldots, u_{2, \ell^{(1)}}^{(1)} \in$ $D_{N^{(1)}+1,2 N^{(1)}}, \ldots, u_{M_{1} 1}^{(1)}, u_{M_{1} 2}^{(1)} \ldots, u_{M_{1} \ell^{(1)}}^{(1)} \in D_{\left(M_{1}-1\right) N^{(1)}+1, M_{1} N^{(1)}}$ satisfying $\left(d_{1}\right)^{\alpha_{1}+\delta_{1}} \leq p_{u_{j l}^{(1)}} \leq\left(d_{1}\right)^{\alpha_{1}-\delta_{1}},\left(d_{1}\right)^{1+\delta_{1}} \leq r_{u_{j l}^{(1)}} \leq$ $\left(d_{1}\right)^{1-\delta_{1}}$ for all $1 \leq j \leq M_{1} ; 1 \leq l \leq \ell^{(1)}$ and $\ell^{(1)} \geq$ $\left(d_{1}\right)^{-\beta^{*}\left(\alpha_{1}\right)+\delta_{1}(q+1)}$.

For $1 \leq j \leq M_{1}$, remark $\mathbf{B}_{j}^{(1)}=\left\{u_{j 1}^{(1)}, u_{j 2}^{(1)}, \ldots, u_{j \ell^{(1)}}^{(1)}\right\}$.

By the same step, for $\alpha_{i}$ and $\delta_{i}$, we also construct $\mathbf{B}_{j}^{(i)}=$ $\left\{u_{j 1}^{(i)}, u_{j 2}^{(i)} \ldots, u_{j e^{(i)}}^{(i)}\right\}\left(1 \leq j \leq M_{i}\right)$ such that

(a) $\ell^{(i)} \geq\left(d_{i}\right)^{-\beta^{*}\left(\alpha_{i}\right)+\delta_{i}(q+1)}$;

(b) $u_{j 1}^{(i)}, u_{j 2}^{(i)}, \ldots, u_{j, \ell^{(i)}}^{(i)} \in D_{\sum_{k=1}^{i-1} M_{i} N^{(k)}+(j-1) N^{(i)}+1, \sum_{k=1}^{i-1} M_{i} N^{(k)}+j N^{(i)}}$;

(c) $d_{i}^{1+\delta_{i}} \leq r_{u_{j l}^{(i)}} \leq d_{i}^{1-\delta_{i}}$ for $1 \leq j \leq M_{i}, 1 \leq l \leq \ell^{(i)}$;

(d) $d_{i}^{\alpha_{i}+\delta_{i}} \leq p_{u_{j l}^{(i)}} \leq d_{i}^{\alpha_{i}-\delta_{i}}$ for $1 \leq j \leq M_{i}, 1 \leq l \leq \ell^{(i)}$.

Also we let $\left\{M_{i}\right\}_{i=1}^{\infty}$ be a sequence of integers large enough such that

(e) $M_{i} N^{(i)}>n_{0}^{(i)}$

(f) $d_{i}^{M_{i}}<\left(d_{i+1}\right)^{2^{i}}$ for each $i \in \mathbb{N}$;

(g) $\lim _{k \rightarrow \infty}\left(\sum_{i=1}^{k} M_{i} \log d_{i} / M_{k+1} \log d_{k+1}\right)=0$.

Now we define a sequence of subsets of symbol set $\Sigma^{*}$ in the following manner:

$$
\mathbf{B}_{1}^{(1)}, \ldots, \mathbf{B}_{M_{1}}^{(1)}, \mathbf{B}_{1}^{(2)}, \ldots, \mathbf{B}_{M_{2}}^{(2)}, \ldots, \mathbf{B}_{1}^{(i)}, \ldots, \mathbf{B}_{M_{i}}^{(i)}, \ldots
$$

and relabel them as $\left\{\mathbf{B}_{n}^{*}\right\}_{n=1}^{\infty}$. Let

$$
F_{q_{1}, q_{2}}=\bigcap_{n=1}^{\infty} \bigcup_{v_{1} \in B_{1}^{*}, \ldots, v_{n} \in B_{n}^{*}} J_{v_{1} v_{2} \cdots v_{n}} .
$$

It is easy to check that $F_{q_{1}, q_{2}}$ is the homogeneous Moran set which is a subset of the homogeneous Moran set $E(\Omega)$.
Next we show that

$$
\begin{gathered}
\liminf _{r \rightarrow 0} \frac{\log \mu(B(x, r))}{\log r}=\beta^{\prime}\left(q_{1}\right), \\
\limsup _{r \rightarrow 0} \frac{\log \mu(B(x, r))}{\log r}=\beta^{\prime}\left(q_{2}\right), \quad \forall x \in F_{q_{1}, q_{2}}, \\
\operatorname{dim}_{H} F_{q_{1}, q_{2}} \geq \beta^{*}\left(\beta^{\prime}\left(q_{1}\right)\right) .
\end{gathered}
$$
that

Let $x \in F_{q_{1}, q_{2}}$, then there exist $v_{i} \in \mathbf{B}_{i}^{*}(i=1,2, \ldots)$ such

$$
\{x\}=\lim _{n \rightarrow \infty} J_{v_{1} \cdots v_{n}} .
$$

Let $n_{k}=M_{1}+M_{2}+\cdots+M_{2 k}-1$; there exists $r_{n_{k}}$ such that

$$
\left|J_{v_{1} \cdots v_{n_{k}+1}}\right| \leq r_{n_{k}}<\left|J_{v_{1} \cdots v_{n_{k}}}\right| .
$$

Using Lemma 10, we attain

$$
\begin{gathered}
\frac{\log p_{v_{1} \cdots v_{n_{k}}}+\Delta_{0} \log \min _{1 \leq i \leq m, 1 \leq j \leq m_{i}}\left\{p_{i, j}\right\}}{\log r_{v_{1} \cdots v_{n_{k}+1}}} \\
\leq \frac{\log \mu\left(B\left(x, r_{n_{k}}\right)\right)}{\log r_{n_{k}}} \\
\leq \frac{\log p_{v_{1} \cdots v_{n_{k}+1}}}{\log r_{v_{1} \cdots v_{n_{k}}}} .
\end{gathered}
$$

Thus to calculate $\lim _{k \rightarrow \infty}\left(\log \mu\left(B\left(x, r_{n_{k}}\right)\right) / \log r_{n_{k}}\right)$, we need to estimate $p_{v_{1} \cdots v_{M}}$ and $r_{v_{1} \cdots v_{M}}$ for $M=n_{k}, n_{k}+1$. By (c) and (d), we obtain

$$
\begin{aligned}
& \prod_{i=1}^{2 k-1} d_{i}^{M_{i}\left(\alpha_{i}+\delta_{i}\right)} d_{2 k}^{M_{2 k}\left(\alpha_{2 k}+\delta_{2 k}\right)} \\
& \leq p_{v_{1} \cdots v_{n_{k}+1}} \leq p_{v_{1} \cdots v_{n_{k}}} \leq \prod_{i=1}^{2 k-1} d_{i}^{M_{i}\left(\alpha_{i}-\delta_{i}\right)} d_{2 k}^{\left(M_{2 k}-1\right)\left(\alpha_{2 k}-\delta_{2 k}\right)}, \\
& \prod_{i=1}^{2 k-1} d_{i}^{M_{i}\left(1+\delta_{i}\right)} d_{2 k}^{M_{2 k}\left(1+\delta_{2 k}\right)} \\
& \leq r_{v_{1} \cdots v_{n_{k}+1}} \leq r_{v_{1} \cdots v_{n_{k}}} \leq \prod_{i=1}^{2 k-1} d_{i}^{M_{i}\left(1-\delta_{i}\right)} d_{2 k}^{\left(M_{2 k}-1\right)\left(1-\delta_{2 k}\right)} .
\end{aligned}
$$

By (54), (60)-(62), and (g), we obtain

$$
\lim _{k \rightarrow \infty} \frac{\log \mu\left(B\left(x, r_{n_{k}}\right)\right)}{\log r_{n_{k}}}=\beta^{\prime}\left(q_{2}\right) .
$$

Let $n_{k}^{\prime}=M_{1}+M_{2}+\cdots+M_{2 k+1}-1$; there exists $r_{n_{k}^{\prime}}$ such that

$$
\left|J_{v_{1} \cdots v_{n_{k}^{\prime}+1}}\right| \leq r_{n_{k}^{\prime}}<\left|J_{v_{1} \cdots v_{n_{k}^{\prime}}}\right|
$$


By the same method, we obtain

$$
\lim _{k \rightarrow \infty} \frac{\log \mu\left(B\left(x, r_{n_{k}^{\prime}}\right)\right)}{\log r_{n_{k}^{\prime}}}=\beta^{\prime}\left(q_{1}\right) .
$$

Next we show $\beta^{\prime}\left(q_{1}\right) \leq \liminf _{r \rightarrow 0}(\log \mu(B(x, r)) /$ $\log (r))<\lim \sup _{r \rightarrow 0}(\log \mu(B(x, r)) / \log (r)) \leq \beta^{\prime}\left(q_{2}\right)$. For $r>0$ small enough, there is a unique large integer $n$ such that

$$
\left|J_{v_{1} \cdots v_{n+1}}\right| \leq r<\left|J_{v_{1} \cdots v_{n}}\right|
$$

Using the same method as above, we attain

$$
\begin{gathered}
\frac{\log p_{v_{1} \cdots v_{n}}+\Delta_{0} \log \min _{1 \leq i \leq m, 1 \leq j \leq m_{i}}\left\{p_{i, j}\right\}}{\log r_{v_{1} \cdots v_{n+1}}} \\
\leq \frac{\log \mu(B(x, r))}{\log r} \\
\leq \frac{\log p_{v_{1} \cdots v_{n+1}}}{\log r_{v_{1} \cdots v_{n}}} .
\end{gathered}
$$

Now we estimate $p_{v_{1} \cdots v_{M}}$ and $r_{v_{1} \cdots v_{M}}$ for $M=n, n+1$. For large $n$, write $n=\sum_{i=1}^{k} M_{i}+p$ with $1 \leq p \leq M_{k+1}$. In the case that $1 \leq p \leq M_{k+1}-1$, by (c) and (d), we obtain

$$
\begin{aligned}
& \prod_{i=1}^{k} d_{i}^{M_{i}\left(\alpha_{i}+3 \delta_{i}\right)} d_{k+1}^{(p+1)\left(\alpha_{k+1}+\delta_{k+1}\right)} \\
& \leq p_{v_{1} \cdots v_{n+1}} \leq p_{v_{1} \cdots v_{n}} \leq \prod_{i=1}^{k} d_{i}^{M_{i}\left(\alpha_{i}-\delta_{i}\right)} d_{k+1}^{p\left(\alpha_{k+1}-\delta_{k+1}\right)}, \\
& \prod_{i=1}^{k} d_{i}^{M_{i}\left(1+\delta_{i}\right)} d_{k+1}^{(p+1)\left(1+\delta_{k+1}\right)} \\
& \leq r_{v_{1} \cdots v_{n+1}} \leq r_{v_{1} \cdots v_{n}} \leq \prod_{i=1}^{k} d_{i}^{M_{i}\left(1-\delta_{i}\right)} d_{k+1}^{p\left(1-\delta_{k+1}\right)} .
\end{aligned}
$$

In the other case $p=M_{k+1}$, we have the similar inequalities where the lower bounds for $p_{v_{1} \cdots v_{n}}$ and $r_{v_{1} \cdots v_{n}}$ in (68) and (69) are replaced, respectively, by

$$
\begin{aligned}
\left(\prod_{i=1}^{k+1} d_{i}^{M_{i}\left(\alpha_{i}+\delta_{i}\right)}\right) d_{k+2}^{\left(\alpha_{k+2}+\delta_{k+2}\right)} & \leq p_{v_{1} \cdots v_{n+1}} \\
& <p_{v_{1} \cdots v_{n}} \leq \prod_{i=1}^{k+1} d_{i}^{M_{i}\left(\alpha_{i}+\delta_{i}\right)}, \\
\left(\prod_{i=1}^{k+1} d_{i}^{M_{i}\left(1+\delta_{i}\right)}\right) d_{k+2}^{\left(1+\delta_{k+2}\right)} & \leq r_{v_{1} \cdots v_{n+1}} \\
& <r_{v_{1} \cdots v_{n}} \leq \prod_{i=1}^{k+1} d_{i}^{M_{i}\left(1+\delta_{i}\right)} .
\end{aligned}
$$

By (54), using the inequalities (67)-(71), (f), and (g), we obtain

$$
\begin{aligned}
\beta^{\prime}\left(q_{1}\right) & \leq \liminf _{r \rightarrow 0} \frac{\log \mu(B(x, r))}{\log r} \\
& <\limsup _{r \rightarrow 0} \frac{\log \mu(B(x, r))}{\log r} \leq \beta^{\prime}\left(q_{2}\right) .
\end{aligned}
$$

Combining (63), (65), and (72), we obtain

$$
\begin{gathered}
\liminf _{r \rightarrow 0} \frac{\log \mu(B(x, r))}{\log r}=\beta^{\prime}\left(q_{1}\right), \\
\limsup _{r \rightarrow 0} \frac{\log \mu(B(x, r))}{\log r}=\beta^{\prime}\left(q_{2}\right), \quad \forall x \in F_{q_{1}, q_{2}} .
\end{gathered}
$$

To prove $\operatorname{dim}_{H} F_{q_{1}, q_{2}} \geq \beta^{*}\left(q_{1}\right)$, recall that $F_{q_{1}, q_{2}}$ is the homogeneous Moran set which is a subset of the homogeneous Moran set $E(\Omega)$. For large $n$, write $n=\sum_{i=1}^{k} M_{i}+p$ with $1 \leq p \leq M_{k+1}$. By (a) and (c), we have

$$
\prod_{s=1}^{n} \# \mathbf{B}_{s}^{*} \geq \prod_{i=1}^{k} d_{i}^{M_{i}\left(-\beta^{*}\left(\alpha_{i}\right)+\delta_{i}\left(q_{i}+1\right)\right)} d_{k+1}^{p\left(-\beta^{*}\left(\alpha_{k+1}\right)+\delta_{k+1}\left(q_{k+1}+1\right)\right)}
$$

and for any $v_{1} \in \mathbf{B}_{1}^{*}, \ldots, v_{n} \in \mathbf{B}_{n}^{*}$

$$
r_{v_{n}} \geq d_{k+1}^{1+\delta_{k+1}}, \quad r_{v_{1} \cdots v_{n}} \leq \sum_{i=1}^{k} d_{i}^{M_{i}\left(1-\delta_{i}\right)} d_{k+1}^{p\left(1-\delta_{k+1}\right)}
$$

Using (75) and (f), we have

$$
\lim _{n \rightarrow \infty} \frac{\log r_{v_{n}}}{\log r_{v_{1} \cdots v_{n}}}=0
$$

This implies the condition (5) in Proposition 3; we have $\operatorname{dim}_{H} F_{q_{1}, q_{2}}=\liminf _{n \rightarrow \infty} s_{n}$, where $s_{n}$ satisfies the equation

$$
\sum_{v_{1} \in B_{1}^{*} \cdots v_{n} \in B_{n}^{*}}\left(r_{v_{1} \cdots v_{n}}\right)^{s_{n}}=1
$$

It follows that

$$
\operatorname{dim}_{H} F_{q_{1}, q_{2}} \geq \liminf _{n \rightarrow \infty} \frac{\log \left(\prod_{s=1}^{n} \# B_{s}^{*}\right)}{-\log r_{v_{1} \cdots v_{n}}} .
$$


This, together with (74)-(75), yields

$$
\begin{gathered}
\operatorname{dim}_{H} F_{q_{1}, q_{2}} \\
\begin{aligned}
\liminf _{k \rightarrow \infty}( & \left(\sum_{i=1}^{k} M_{i}\left(\beta^{*}\left(\alpha_{i}\right)-\delta_{i}\left(q_{i}+1\right)\right) \log d_{i}\right. \\
& \left.+p\left(\beta^{*}\left(\alpha_{k+1}\right)-\delta_{k+1}\left(q_{k+1}+1\right)\right) \log d_{k+1}\right)
\end{aligned} \\
\left.\times\left(\sum_{i=1}^{k} M_{i}\left(1-\delta_{i}\right) \log d_{i}+p\left(1-\delta_{k+1}\right) \log d_{k+1}\right)^{-1}\right) \\
\geq \liminf _{k \rightarrow \infty}\left(\left(\sum_{i=1}^{k} M_{i}\left(\beta^{*}\left(\beta^{\prime}\left(q_{1}\right)\right)-\delta_{i}\left(q_{i}+1\right)\right) \log d_{i}\right.\right. \\
\left.+p\left(\beta^{*}\left(\beta^{\prime}\left(q_{1}\right)\right)-\delta_{k+1}\left(q_{k+1}+1\right)\right) \log d_{k+1}\right) \\
\left.\times\left(\sum_{i=1}^{k} M_{i}\left(1-\delta_{i}\right) \log d_{i}+p\left(1-\delta_{k+1}\right) \log d_{k+1}\right)^{-1}\right) \\
\text { since } \left.\beta^{*} \text { is concave }(\operatorname{see}[3])\right) . \\
\geq \beta^{*}\left(\beta^{\prime}\left(q_{1}\right)\right) \\
\text { by }(54) \text { and } \beta^{*}\left(\beta^{\prime}\left(q_{1}\right)\right)<\beta^{*}\left(\beta^{\prime}\left(q_{2}\right)\right)
\end{gathered}
$$

Using Lemma 9, we finish proof of Theorem 7.

\section{Conflict of Interests}

The authors declare that there is no conflict of interests regarding the publication of this paper.

\section{Acknowledgments}

The authors are supported by Natural Science Foundation of China (11271140) and Teaching Research Project of Wuhan College, Zhongnan University of Economics and Law.

\section{References}

[1] D.-J. Feng, K.-S. Lau, and J. Wu, "Ergodic limits on the conformal repellers," Advances in Mathematics, vol. 169, no. 1, pp. 58-91, 2002.

[2] J. E. Hutchinson, "Fractals and self-similarity," Indiana University Mathematics Journal, vol. 30, pp. 713-747, 1981.

[3] M. Wu, "The multifractal spectrum of some Moran measures," Science in China A: Mathematics, vol. 48, no. 8, pp. 1097-1112, 2005.

[4] Y. Peres and B. Solomyak, "Existence of $L^{q}$ dimensions and entropy dimension for self-conformal measures," Indiana University Mathematics Journal, vol. 49, no. 4, pp. 1603-1621, 2000.
[5] J. Xiao and M. Wu, "The multifractal dimension functions of homogeneous moran measure," Fractals, vol. 16, no. 2, pp. 175185, 2008.

[6] L. Barreira and J. Schmeling, "Sets of "non-typical" points have full topological entropy and full Hausdorff dimension," Israel Journal of Mathematics, vol. 116, pp. 29-70, 2000.

[7] E. Chen and J. Xiong, "The pointwise dimension of self-similar measures," Chinese Science Bulletin, vol. 44, no. 23, pp. 21362140, 1999.

[8] L. Olsen and S. Winter, "Normal and non-normal points of self-similar sets and divergence points of self-similar measures," Journal of the London Mathematical Society, vol. 67, no. 1, pp. 103-122, 2003.

[9] J.-Q. Xiao, M. Wu, and F. Gao, "Divergence points of self-similar measures satisfying the OSC," Journal of Mathematical Analysis and Applications, vol. 379, no. 2, pp. 834-841, 2011.

[10] J. Li, M. Wu, and Y. Xiong, "Hausdorff dimensions of the divergence points of self-similar measures with the open set condition," Nonlinearity, vol. 25, no. 1, pp. 93-105, 2012. 


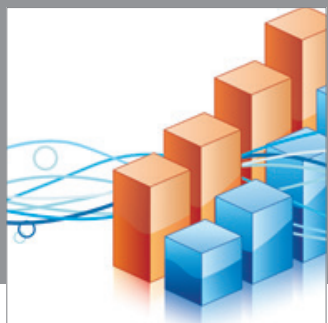

Advances in

Operations Research

mansans

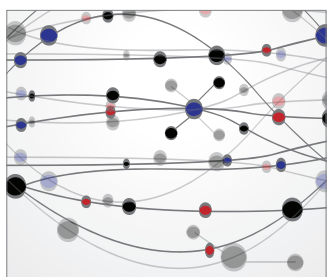

The Scientific World Journal
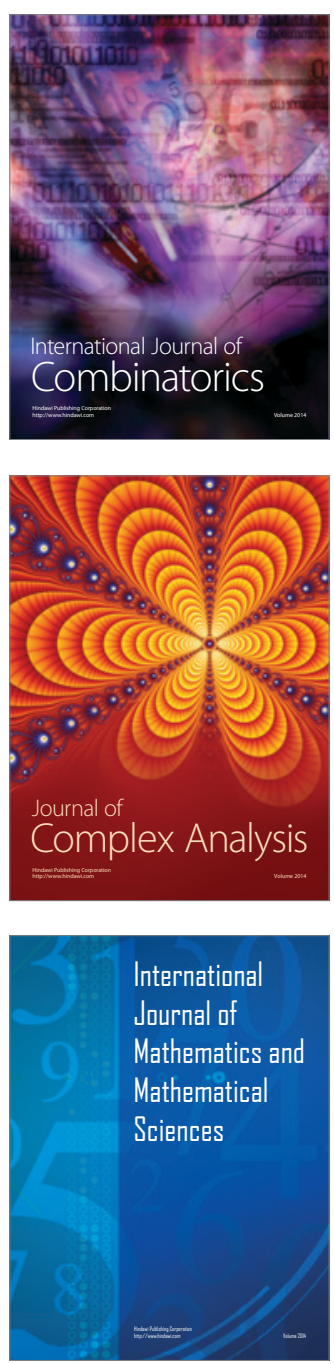
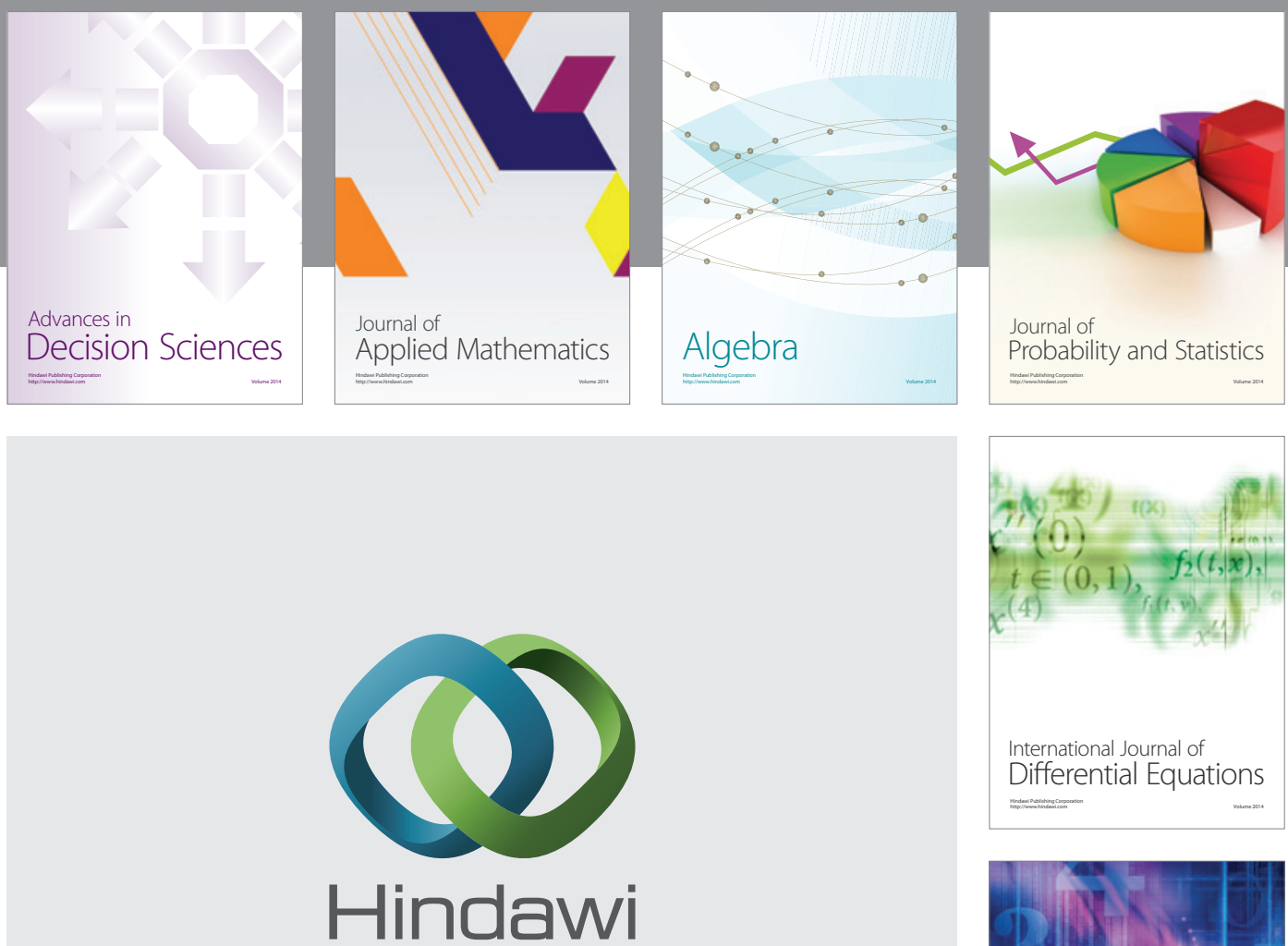

Submit your manuscripts at http://www.hindawi.com
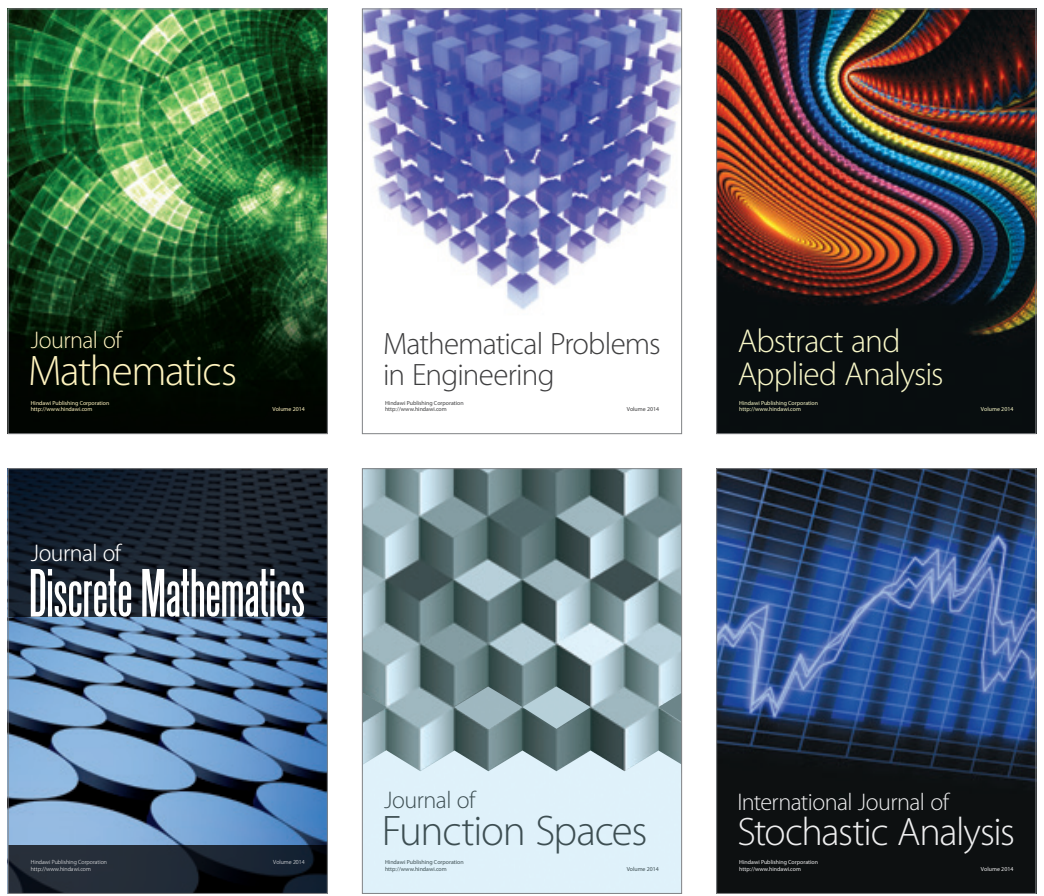

Journal of

Function Spaces

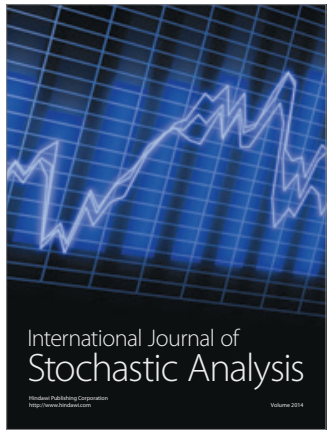

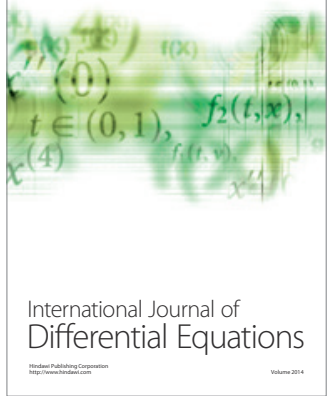
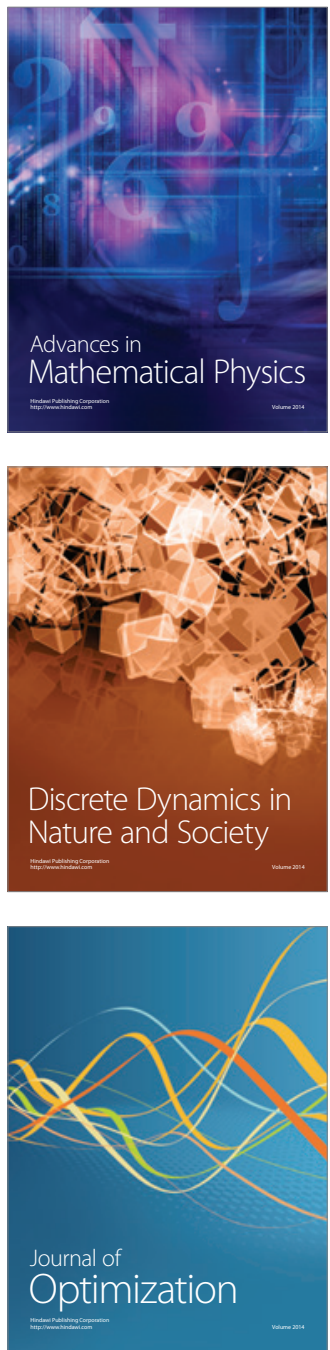